Research Article

\title{
An Analytical Mechanical Model of Corti in the Cochlea
}

\author{
Jiangtao Su $(\mathbb{D}$, Wenjuan Yao $\mathbb{D}$, and Zhengshan Zhao \\ School of Mechanics and Engineering Science, Shanghai University, Shanghai 200444, China \\ Correspondence should be addressed to Wenjuan Yao; wjyao@shu.edu.cn
}

Received 27 May 2020; Revised 11 November 2020; Accepted 17 November 2020; Published 9 December 2020

Academic Editor: Yaobing Zhao

Copyright (c) 2020 Jiangtao Su et al. This is an open access article distributed under the Creative Commons Attribution License, which permits unrestricted use, distribution, and reproduction in any medium, provided the original work is properly cited.

The organ of Corti (OC) in the cochlea is a significant structure for feeling sound. The components of OC and the interaction of the part with the surroundings contribute to the fact that the passive tuning of the cochlear macrostructure is unclear. Based on the interaction between the basilar membrane (BM), tectorial membrane (TM), reticular lamina (RL), and various parts of OC, a mechanical model of the cochlea is established to study the motion patterns of each part under the action of a certain pressure. The variational principle is applied to the calculation of the analytical expression of the displacement of the $\mathrm{BM}$. The results of the analytical solution differ little from the experimental value, and the variation trend is consistent, which presents the correctness of the model. The parameter sensitivity analysis is carried out for obtaining the interaction principle and the primary and secondary roles of each component in the process of the sense of sound. The results show that the absence of the TM and the decrease in the stiffness of the outer hair cells (OHCs) and OHC bundles will shift vibratory response patterns to lower frequencies, in which the lack of TM will result in the greatest reduction of CF. The absence of RL exerts a negative influence on the CF as well as the amplitude of BM and thereby loss of hearing. Therefore, both TM and RL are essential structures during the process of the sense of sound. At the same time, the resonance frequency at the base of the $\mathrm{BM}$ is concentrated on the high-frequency segment, while the apex of the BM is mainly in the low frequency. Different points of BM correspond to different $\mathrm{CF}$, which demonstrates the frequency selectivity of the BM.

\section{Introduction}

As a part of the section of the cochlea, OC plays a significant role in the process of the sense of sound. The incoming vibration from the middle ear will cause sound pressure waves in the inner ear, thereby moving the $\mathrm{OC}$ relative to surrounding structures. The relative motion will cause the impulse of the auditory nerve, which is the key to producing hearing in the brain. With the tuning of OC, the amplitude of the BM can increase vibratory magnitudes by 1000 times of the original value [1]. How does this remarkable acoustic behavior come about?

The reasons for this phenomenon are thought to be dominated by the active effect of OHCs [2-4]. However, the contributions from the various components of OC itself could not be excluded [5]. So far, scientists have not been able to accurately interpret the sound-sensing mechanism of the cochlea. The effect of various components of OC on the passive tuning of the cochlea is also unclear.
In the meantime, noninvasive measurement of the motion inside OC is very inconvenient. Therefore, cochlear mechanics has entered the stage of exploring the soundsensing mechanism of the cochlea and has become a research hotspot in this century. However, the physical properties of each part of OC are unknown. Consequently, in the early stage, many experts and scholars focused on the structural parameters and material properties of the OC. Fernandez [6] measured the length, width, and thickness of the $\mathrm{BM}$ of the guinea pig cochlea with a calibration eyepiece. Edge et al. [7] observed the structure of living cochlea from a radial perspective for the first time and obtained the length and thickness of OHCs at different points of BM. Glueckert et al. [8] measured the length and thickness of OHC bundles using high-power scanning electron microscopy. Nilsen and Russell [9] obtained the relative position relationship of various parts of $\mathrm{OC}$ in guinea pigs through histological sections. Sugawara et al. [10] used atomic force microscopy to measure the local mechanical properties of guinea pig 
OHCs. Zagadou et al. [11] obtained the elastic modulus of $\mathrm{OHC}$ bundles and BM by the method of point-stiffness measurements. Cai et al. [12] used the formula to convert the measured stiffness of OHCs into the elastic modulus of OHCs. With further study, Cooper [13] and Nilse and Russell [14] found that the vibration of the center of the BM was larger than the vibration at both ends. TM and RL also have the largest amplitude in the middle $[15,16]$. At the same time, some scholars believe that the modes of the cochlear partition are not single during the vibration process [17]. These experimental data and analytical studies provide a theoretical basis for the development of the cochlear mechanics model.

For the study of the model, many scholars have focused on the interaction between the lymph fluid and the BM at the macrostructural level of the cochlea. In the early stage, researchers generally spread the spiral cochlea into a straight cochlea for analysis. Allen [18] established a 2D cochlea mechanical model and greatly simplified the model, and the cochlear interface was assumed to be rectangular. After that, Mammano and Nobili [19] built a 2D model considering the variation of cochlea cross section. Steele and Taber [20] established a 3D linear cochlear mechanical model, which included fluid action, and analyzed the mechanical behavior of the cochlea using the WKB method. However, with the development of research, it is found that the spiral shape of the cochlea may not only improve the space utilization of the cochlea [21, 22]. Recent experiments [23, 24] have demonstrated that the spiral shape of the cochlea can enhance the hearing ability of low-frequency signals in the cochlea. Therefore, some scholars gradually began to establish spiral cochlea for analysis. Lieberstein [25, 26] established a cochlea with a spiral shape BM but did not consider the fluidsolid coupling between the BM and lymph. Ma and Yao [27] built a 3D spiral cochlea to study the flow field distribution of lymph fluid in the cochlea and the vibration of BM. At the same time, many scholars used numerical methods to study the mechanical behavior of the cochlea. Gan et al. [28] first built a complete cochlea model containing a linear cochlea, which was applied to study the mechanical behaviors of BM. Later, Wang et al. [29] established a cochlea model containing a linear cochlea and combined it with the active feedforward mechanism to analyze the nonlinear vibration performance of the active cochlea. To make the finite element model closer to reality, Givelberg and Bunn [30] established a spiral finite element model of the cochlea and used the model to reproduce some known features of the cochlea mechanics. After that, in the study of implantable hearing devices, Gan established a spiral cochlear model [31], which can more intuitively analyze the implanted hearing equipment and the operation process. Yao et al. [32-35] built a 3D model of the cochlea with a spiral shape to describe the fluid-solid coupling motion of the lymphatic fluid and BM.

However, these models assumed that the motion of each part in OC is consistent with the movement of the BM and ignored the relative motion of the RL, the TM, and so forth during the vibration process. Moreover, the stiffness of the
$\mathrm{RL}$ is far greater than that of the BM. The effect of the RL is essential for the interaction of the structures of OC. Therefore, in this paper, a cochlear mechanical model containing all parts of the OC was established according to the relative motion relations among the BM, OHCs, RL, and TM. Through parameter sensitivity analysis, we mainly explored the role of RL in the cochlear sensory mechanism and the effect of the RL on the passive tuning of OC. At the same time, the role of OHC bundles, OHCs, and TM in the passive mechanism of the cochlea was also studied.

\section{Model}

2.1. Establishment of a Mechanical Model. The mechanical model of OC was established based on the medical anatomical diagram of the guinea pig cochlea (Figure 1) and the geometric relationship of each part in OC. The mechanical model of OC includes RL, TM, OHCs, and OHC bundles. Both ends of the BM are fixed constraints. The Deiters cells are considered as a rigid connection due to their high stiffness. The arch of OC composed of outer pillar cell and inner pillar cell is assumed to be a rigid structure. There are three $\mathrm{OHC}$ bundles on the RL. The bottom of the OHC bundles connects with the top of the OHC. The top of the OHC bundles is embedded in the TM to form a fixed constraint. The $\mathrm{OHC}$ bundles can deform with vibration. The part of TM near the cochlear pivot is a fixed connection. The model does not include the motion of TM because of its high quality and rigidity. The mechanical model of $\mathrm{OC}$ is shown in Figure 2.

2.2. Displacement Relationships of Each Point of OC. In the OC structure, displacement of the BM can express that of the OHCs, OHC bundles, RL, and the arch of OC.

The mode of BM vibrations is assumed to be sinusoidal vibration. When the calculation point is $x$ and the time is $t$, the displacement of the BM of Cochlea section is given by

$$
u_{b m}(x, y, t)=A \sin (\pi y / b),
$$

In the formula, $y$ represents the distance between the calculated point and the left end of the BM, $b$ is the width of the BM, $x$ is the distance between the selected point and the basal of the BM, and $A$ represents the vibration amplitude of the BM.

The arch structure is assumed to be rigid. When the BM is displaced, the arch structure will rotate in response. The displacement of the apex of the arch structure is given by

$$
u_{o c}=A \sin \left(\pi L_{p c} / b\right) 2 \cos \beta
$$

In the formula, $L_{p c}$ is the distance between the left end and the right end of the arch structure and $\beta$ is the inner angle of the arch structure.

The compression deformation of the OHCs depends on the relative displacement of the top and the bottom of the $\mathrm{OHCs}$. The motion of the top of the OHCs in the middle row is given by 


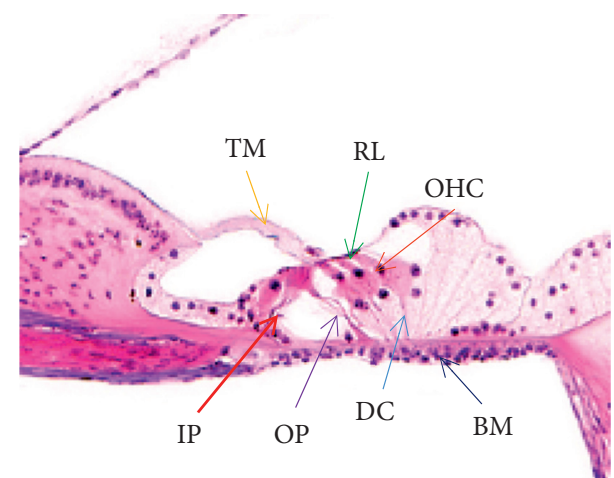

Figure 1: Cross-sectional anatomy of guinea pig [36]. IP, inner pillar cell; OP, outer pillar cell; DC, Deiters cell; BM, basilar membrane; OHCs, outer hair cells; RL, reticular lamina; TM, tectorial membrane.

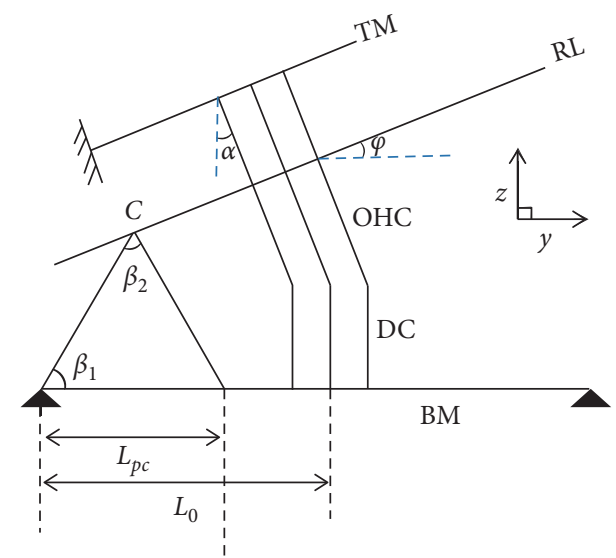

Figure 2: Micromechanical model of OC structure. $L_{p c}$ is the distance between the left end and the right end of the arch structure, $L_{0}$ is the distance between the bottom of the middle row of OHCs and the left end of the BM, $\phi$ is the acute angle between $\mathrm{OHCs}$ and $z$-axis, $\alpha$ is the acute angle between OHCs bundles and $z$-axis, $\alpha$ and $\phi$ have the same value, and $\beta_{1}=\beta_{2}=\beta$ is the inside angle of the arch structure.

$$
u_{\text {ohc-middle }}^{a}=A \sin \left(\pi L_{p c} / b\right) 2 \cos \beta[-\cos (\beta-\phi)+\cos (\beta-\alpha)] \text {, }
$$

where $\phi$ is the acute angle between OHCs and the $z$-axis and $\alpha$ is the acute angle between OHCs bundles and the $z$-axis.

The displacement of the bottom of the middle row of the OHCs is

$$
u_{\text {ohc-middle }}^{b}=A \cos \phi \text {, }
$$

The shear displacement of the OHC bundles in the middle row is

$$
u_{h b-\text { middle }}=A \sin \left(\pi L_{p c} / b\right) 2 \cos \beta \sin (\beta-\phi) .
$$

There was no significant difference in the displacement of three rows of OHCs. Their values are considered to be the same.

2.3. Analytical Solution of BM. The work done by external forces is mainly absorbed by the movement of $\mathrm{BM}, \mathrm{OHCs}$, and OHC bundles. When the calculation point is $x$ and the time is $t$, the energy expression of OC components can be obtained according to the content of material mechanics.

The BM is regarded as an isotropic plate [20], and the strain potential energy of the BM is given by

$$
\begin{aligned}
E_{b m}= & \int_{0}^{b} \frac{1}{2 \pi} \int_{0}^{2 \pi} \frac{D}{2}\left[\left(\frac{\partial^{2} u_{b m}}{\partial x^{2}}\right)^{2}+\left(\frac{\partial^{2} u_{b m}}{\partial y^{2}}\right)^{2}\right. \\
& \left.+2 v\left(\frac{\partial^{2} u_{b m}}{\partial x^{2}}\right)\left(\frac{\partial^{2} u_{b m}}{\partial y^{2}}\right)+2(1-v)\left(\frac{\partial^{2} u_{b m}}{\partial x \partial y}\right)^{2}\right] \mathrm{d} \theta \mathrm{d} y,
\end{aligned}
$$

where

$$
\begin{aligned}
& D=E_{1} h_{b m} c^{2}, \\
& c^{2}=\frac{h_{b m}^{2}}{\left[12\left(1-v^{2}\right)\right]},
\end{aligned}
$$

The formula is used to analyze the section of the cochlea, and the integration formula of the strain energy of the BM is

$$
E_{b m}=\frac{\pi^{4}}{2 b^{3}} \frac{E_{1} h_{b m}^{3}}{24\left(1-v^{2}\right)} A^{2} h_{o h c},
$$

where $E_{1}$ is the elastic modulus of the BM, $u_{b m}$ is the displacement of the $\mathrm{BM}, h_{b m}$ is the thickness of the $\mathrm{BM}, h_{o h c}$ is the thickness of the OHCs, and $v$ is Poisson's ratio of the BM.

The axial deformation of $\mathrm{OHC}$ depends on the relative displacement of the top and bottom of OHC. The strain energy generated by OHCs is

$$
\begin{aligned}
& E_{\text {ohc }}=3 \times \frac{1}{2} F_{o h c} u_{o h c}, \\
& F_{o h c}=\frac{u_{o h c} E_{2} A_{o h c}}{L_{o h c}},
\end{aligned}
$$

and the integration formula of the strain energy of the OHCs is 


$$
\begin{aligned}
& E_{o h c}=\frac{3}{2} \times \frac{E_{2} A_{o h c} u_{o h c}^{2}}{L_{o h c}} \\
& u_{o h c}=\left|A \sin \left(\pi L_{p c} / b\right) 2 \cos \beta[\cos (\beta-\alpha)-\cos (\beta-\phi)]-A \cos \phi\right| \\
& E_{o h c}=\frac{3}{2} \times \frac{E_{2} A_{o h c}\left(\sin \left(\pi L_{p c} / b\right) 2 \cos \beta[\cos (\beta-\alpha)-\cos (\beta-\phi)]-\cos \phi\right)^{2} A^{2}}{L_{o h c}}, \\
& \qquad T_{o h c}=\frac{3}{8} \rho_{o h c} L_{o h c} h_{o h c}^{2}\left[\cos \phi+2 \sin \left(\pi L_{p c} / b\right) \cos \beta(-\cos (\beta-\phi)\right. \\
& \text { modulus of OHCs, } A_{o h c} \text { is the cross- } \\
& L_{\text {ohc }} \text { is the length of OHCs, and } u_{\text {ohc }} \\
& \quad+\cos (\beta-\alpha))]^{2}(w A)^{2}, \\
& \text { of OHCs. }
\end{aligned}
$$

where $E_{2}$ is the elastic modulus of OHCs, $A_{\text {ohc }}$ is the crosssectional area of OHCs, $L_{o h c}$ is the length of OHCs, and $u_{o h c}$ is the axial deformation of OHCs.

The strain energy equations generated by $\mathrm{OHC}$ bundles are

$$
\begin{aligned}
E_{\text {ste }} & =3 \times 6 E_{3} I_{s t e} u_{s t e}^{2} / L_{s t e}^{3}, \\
I_{\text {ste }} & =L_{s t e} h_{s t e}^{3} / 12, \\
u_{s t e} & =u_{h b \text {-middle }}=A \sin \left(\pi L_{p c} / b\right) 2 \cos \beta \sin (\beta-\phi),
\end{aligned}
$$

and the integration formula of the strain energy of the $\mathrm{OHC}$ bundles is

$$
E_{\text {ste }}=\frac{6 E_{3} h_{s t e}^{3} \sin ^{2}\left(\pi L_{p c} / b\right) \cos ^{2} \beta \sin ^{2}(\beta-\phi) A^{2}}{L_{s t e}^{2}},
$$

where $E_{3}$ is the elastic modulus of the OHC bundles, $h_{\text {ste }}$ is the thickness of the OHC bundles, $L_{s t e}$ is the length of the OHC bundles, and $u_{s t e}$ is the shear displacement perpendicular to the axis of $\mathrm{OHC}$ bundles.

The total kinetic energy generated by $\mathrm{BM}$ is given by

$$
\begin{aligned}
T_{b m} & =\frac{1}{2} m_{b m} v_{b m}^{2}=\int_{0}^{b} \frac{1}{2} \rho_{b m} h_{b m} b\left[w A \sin \left(\frac{\pi y}{b}\right)\right]^{2} d y h_{o h c} \\
& =\frac{b^{2}}{4} \rho_{b m} h_{b m}(w A)^{2} h_{o h c},
\end{aligned}
$$

where $m_{b m}$ is the mass of the BM, $v_{b m}$ is the velocity of the $\mathrm{BM}, \rho_{b m}$ is the density of the BM, and $w$ is the angular frequency.

The kinetic energy generated by OHCs is given by

$$
\begin{aligned}
T_{o h c}= & \frac{1}{2} m_{o h c} v_{o h c}^{2}=3 \times \frac{1}{2} \times \rho_{o h c} L_{o h c} h_{o h c}\left(w \bar{u}_{o h c}\right)^{2} h_{o h c}, \\
\bar{u}_{o h c}= & \frac{1}{2}\left(A \cos \phi+A \sin \left(\pi L_{p c} / b\right) 2 \cos \beta[-\cos (\beta-\phi)\right. \\
& +\cos (\beta-\alpha)])
\end{aligned}
$$

and the integration formula of the kinetic energy of the $\mathrm{OHCs}$ is where $\rho_{\text {ohc }}$ is the density of OHCs, $m_{\text {ohc }}$ is the mass of OHCs, $\bar{u}_{\text {ohc }}$ is the average displacement of OHCs, and $v_{\text {ohc }}$ is the speed of OHCs.

The kinetic energy generated by $\mathrm{OHC}$ bundles is given by

$$
\begin{aligned}
& T_{s t e}=\frac{1}{2} m_{s t e} v_{s t e}^{2}=3 \times \frac{1}{2} \rho_{s t e} L_{s t e} h_{s t e}\left(w \bar{u}_{s t e}\right)^{2} h_{s t e}, \\
& \bar{u}_{s t e}=\frac{1}{2} A \sin \left(\pi L_{p c} / b\right) 2 \cos \beta \sin (\beta-\phi),
\end{aligned}
$$

and the integration formula of the kinetic energy of the $\mathrm{OHC}$ bundles is

$$
T_{s t e}=\frac{3}{2} \rho_{s t e} L_{s t e} h_{s t e}^{2}\left(\sin \left(\pi L_{p c} / b\right) \cos \beta \sin (\beta-\phi)\right)^{2}(w A)^{2}
$$

where $m_{\text {ste }}$ is the mass of the OHC bundles, $v_{\text {ste }}$ is the velocity of the OHC bundles, $\rho_{\text {ste }}$ is the density of the OHC bundles, and $\bar{u}_{s t e}$ is the average displacement of the $\mathrm{OHC}$ bundles.

The energy equation $W$ of external force $P$ is given by

$$
W=\int_{0}^{b} P \sin (w t) A \sin \left(\frac{\pi y}{b}\right) d y h_{o h c}=\frac{2 b}{\pi} P A \sin (w t) h_{o h c}
$$

the structural strain energy $V$ is given by

$$
V=E_{b m}+E_{o h c}+E_{s t e}-W
$$

and the total potential energy of OC is given by

$$
U=V-T=E_{b m}+E_{o h c}+E_{s t e}-W-T_{b m}-T_{o h c}-T_{s t e},
$$

By substituting the expressions of each term into equation (20), the total potential energy can be obtained from the following equation: 


$$
\begin{aligned}
U= & \frac{3}{2} \times \frac{E_{2} A_{o h c}\left(\sin \left(\pi L_{p c} l b\right) 2 \cos \beta[\cos (\beta-\alpha)-\cos (\beta-\phi)]-\cos \phi\right)^{2} A^{2}}{L_{o h c}}+\frac{6 E_{3} h_{s t e}^{3} \sin ^{2}\left(\pi L_{p c} / b\right) \cos ^{2} \beta \sin ^{2}(\beta-\phi) A^{2}}{L_{s t e}^{2}} \\
& +\frac{\pi^{4}}{2 b^{3}} \frac{E_{1} h_{b m}^{3}}{24\left(1-v^{2}\right)} A^{2} h_{o h c}-\frac{2 b}{\pi} P A \sin (w t) h_{o h c}-\frac{b^{2}}{4} \rho_{b m} h_{b m} w^{2} h_{\text {ohc }} A^{2}-\frac{3}{2} \rho_{s t e} L_{s t e} h_{s t e}^{2}\left(\sin \left(\pi L_{p c} / b\right) \cos \beta \sin (\beta-\phi)\right)^{2} w^{2} A^{2}- \\
& -\frac{3}{8} \rho_{\text {ohc }} L_{\text {ohc }} h_{\text {ohc }}^{2}\left[\cos \phi+2 \sin \left(\pi L_{p c} / b\right) \cos \beta(-\cos (\beta-\phi)+\cos (\beta-\alpha))\right]^{2} w^{2} A^{2} .
\end{aligned}
$$

The first-order variation of $A$ is

$$
\delta U=0,
$$

When the calculation point is $x$ and the time is $t$, the expression of BM amplitude is as follows:

$$
\begin{aligned}
A= & \frac{(2 b / \pi) P \sin (w t) h_{o h c}}{\pi^{4} E_{1} h_{b m}^{3} h_{o h c} / 24 b^{3}\left(1-v^{2}\right)+3 E_{2} A_{o h c}\left(\sin \left(\pi L_{p c} / b\right) 2 \cos \beta[\cos (\beta-\alpha)-\cos (\beta-\varphi)]-\cos \varphi\right)^{2} / L_{o h c}} \\
& +\left(12 E_{3} h_{s t e}^{3} \sin ^{2}\left(\pi L_{p c} / b\right) \cos ^{2} \beta \sin ^{2}(\beta-\varphi) / L_{s t e}^{2}\right)-3 \rho_{s t e} L_{s t e} h_{s t e}^{2}\left(\sin \left(\pi L_{p c} / b\right) \cos \beta \sin (\beta-\varphi)\right)^{2} w^{2} \\
& -\left(b^{2} / 2\right) \rho_{b m} h_{b m} w^{2} h_{o h c}-(3 / 4) \rho_{o h c} L_{o h c} h_{o h c}^{2}\left[\cos \varphi+2 \sin \left(\pi L_{p c} / b\right) \cos \beta(-\cos (\beta-\varphi)+\cos (\beta-\alpha))\right]^{2} w^{2} .
\end{aligned}
$$

If $A=A_{0} \sin (w t)$, the expression of $A_{0}$ is

$$
\begin{aligned}
A_{0}= & \frac{(2 b / \pi) P h_{o h c}}{\pi^{4} E_{1} h_{b m}^{3} h_{o h c} / 24 b^{3}\left(1-v^{2}\right)+3 E_{2} A_{o h c}\left(\sin \left(\pi L_{p c} / b\right) 2 \cos \beta[\cos (\beta-\alpha)-\cos (\beta-\varphi)]-\cos \varphi\right)^{2} / L_{o h c}} \\
& +\left(12 E_{3} h_{s t e}^{3} \sin ^{2}\left(\pi L_{p c} / b\right) \cos ^{2} \beta \sin ^{2}(\beta-\varphi) / L_{s t e}^{2}\right)-3 \rho_{s t e} L_{s t e} h_{s t e}^{2}\left(\sin \left(\pi L_{p c} / b\right) \cos \beta \sin (\beta-\varphi)\right)^{2} w^{2} \\
& -\left(b^{2} / 2\right) \rho_{b m} h_{b m} w^{2} h_{o h c}-(3 / 4) \rho_{o h c} L_{o h c} h_{o h c}^{2}\left[\cos \varphi+2 \sin \left(\pi L_{p c} / b\right) \cos \beta(-\cos (\beta-\varphi)+\cos (\beta-\alpha))\right]^{2} w^{2} .
\end{aligned}
$$

Therefore, $A_{0}$ is the maximum amplitude of the calculation point in the process of vibration.
Since $w$ is the angular frequency, from $w=2 \pi f$, we can get the maximum amplitude $A_{0}$ expressed by frequency $f$.

$$
\begin{aligned}
A_{0}= & \frac{(2 b / \pi) P h_{\text {ohc }}}{\pi^{4} E_{1} h_{b m}^{3} h_{o h c} / 24 b^{3}\left(1-v^{2}\right)+3 E_{2} A_{\text {ohc }}\left(\sin \left(\pi L_{p c} / b\right) 2 \cos \beta[\cos (\beta-\alpha)-\cos (\beta-\varphi)]-\cos \varphi\right)^{2} / L_{o h c}} \\
& +\left(12 E_{3} h_{s t e}^{3} \sin ^{2}\left(\pi L_{p c} / b\right) \cos ^{2} \beta \sin ^{2}(\beta-\varphi) / L_{s t e}^{2}\right)-12 \pi^{2} \rho_{s t e} L_{s t e} h_{s t e}^{2}\left(\sin \left(\pi L_{p c} / b\right) \cos \beta \sin (\beta-\varphi)\right)^{2} f^{2} \\
& -2 b^{2} \pi^{2} \rho_{b m} h_{b m} f^{2} h_{o h c}-3 \pi^{2} \rho_{o h c} L_{o h c} h_{o h c}^{2}\left[\cos \varphi+2 \sin \left(\pi L_{p c} / b\right) \cos \beta(-\cos (\beta-\varphi)+\cos (\beta-\alpha))\right]^{2} f^{2}
\end{aligned}
$$

In the formula, $P$ is the external force acting on the $\mathrm{BM}$, which is replaced by the uniform distributed force. The pressure difference between the upper and lower parts of the $\mathrm{BM}$ decides the size of uniform pressure [37]. Moreover, the coefficient in equation (25) depends on the selected calculation point except for $f$.

Therefore, when the calculation point on the BM is determined, equation (25) is a function that changes with 
frequency $f$. There is a frequency $f$ that makes $A_{0}$ get the maximum value, which is the $\mathrm{CF}$ of the solution point.

\section{Verification of Model}

3.1. CF of Mammals. By comparison with the experimental data of $\mathrm{CF}$, the accuracy of the analytical solution can be verified. In the early days of cochlear research, Békésy discovered the systematic mapping between the position of the BM and CF. Greenwood [38, 39] also measured several species to obtain the formula relationship of frequencyposition relationship. The tonotopic map satisfies the following formula:

$$
C F=M\left(10^{\alpha x_{0}}-N\right)
$$

where $C F$ is characteristic frequency. The unit of $C F$ is $\mathrm{kHZ}$. $\alpha$ and $N$ are the parameters associated with the selected species, and they have the same value for the same species. The parameter of the expression is mainly from [39]. The value of $\alpha$ is 2.1 and it has small change when choosing different lengths of BM. The value of the parameter $N$ is generally $0.85 . M$ is a constant that varies with the scope of $C F$. The value of gerbil is 0.4 , the guinea pig is 0.35 , and the cat is $0.456 . x_{0}$ is the ratio of the distance between the top of the $\mathrm{BM}$ and the selected position to the total length of the $\mathrm{BM}$, ranging between 0 and 1 .

3.2. Model Parameters. The model was validated with guinea pig cochlea data.

3.2.1. Geometric Parameters. The geometric parameters of each part of the OC are listed in Table 1. The relevant parameters are from [6-10].

According to the presumption that the structural parameters $b, L_{o h c}, h_{b m}, \alpha$, and $\phi$ change linearly along the length of BM, the general form is as follows:

$$
Y=c x+d
$$

where $Y$ is $b, L_{o h c}, h_{b m}, \alpha$, and $\phi$ in Table 1 and $x$ is the distance from the selection point to the bottom of the BM. $c$ and $d$ are the coefficients decided by the geometric parameters of the bottom and the top of the cochlea. The corresponding expressions are given by the following:

The width of BM:

$$
L_{b m}=6.8571 x+62.8573,
$$

the thickness of BM:

$$
h_{b m}=-0.3429 x+7.8572,
$$

the length of OHCs:

$$
L_{o h c}=2.2857 x+64.2858,
$$

and the acute angle:

$$
\alpha=\phi=1.1429 x+22.142 .
$$

3.2.2. Mechanical Properties of Materials. The material properties of the BM, OHCs, and $\mathrm{OHC}$ bundles in OC are shown in Table 2. The relevant parameters are from [10-12, 28].

The parameters $E_{1}, E_{2}$, and $E_{3}$ are the elastic moduli of $\mathrm{BM}, \mathrm{OHCs}$, and $\mathrm{OHC}$ bundles, respectively, which are assumed to change exponentially along the length of BM. The general form can be written as

$$
E=M e^{N x},
$$

where $E$ is the elastic modulus, $M$ and $N$ are the parameters derived from the corresponding data at the top and bottom of the cochlea, and $x$ is the distance from the selection point to the bottom of the BM.

The data of the base and apex of BM, OHCs, and OHC bundles are substituted into the formula as boundary conditions. The elastic modulus function of each part of OC is given by

$$
\begin{aligned}
& E_{1}=3.0663 \times 10^{8} e^{-0.1712 x}, \\
& E_{2}=5.5775 \times 10^{3} e^{-0.086 x}, \\
& E_{3}=1.3889 \times 10^{7} e^{-0.1316 x} .
\end{aligned}
$$

3.3. Validation of the Model. The BM length of the guinea pig in this paper is $25 \mathrm{~mm}$ [5]. According to equation (25), the CF of eight different points $(2.5 \mathrm{~mm}, 5.0 \mathrm{~mm}, 7.5 \mathrm{~mm}, 10 \mathrm{~mm}$, $12.5 \mathrm{~mm}, 15 \mathrm{~mm}, 17.5 \mathrm{~mm}$, and $20 \mathrm{~mm}$ ) of the $\mathrm{BM}$ is calculated.

According to $[38,39]$, the $C F$ of the experimental value is given by

$$
C F=0.35\left(10^{2.1 x_{0}}-0.85\right)
$$

After determining the calculation point on the BM, the experimental value of $C F$ can be obtained by equation (34), which is compared with the analytical value achieved from equation (25), as shown in Figure 3.

Figure 3 illustrates that the calculated analytical value is slightly larger than the experimental value. The slope of the frequency-place map of analytical value is similar to the measured experimental data. The accuracy of the analytic equation is verified. In addition, $C F$ corresponding to different points on the BM is different. At the base, the $C F$ is larger, about $31 \mathrm{kHz}$; at the apex, the $C F$ is smaller, about $900 \mathrm{~Hz}$; this phenomenon is consistent with our understanding of the frequency selectivity of the BM.

Figure 4 shows the frequency response curve of the calculation point. The displacement of the BM in Figure 4 represents the absolute value. Each response curve has a different peak. Moreover, the resonance frequency at the base of the BM is concentrated in the high-frequency segment, while the apex of the BM is mainly in the low frequency. Different points correspond to different $C F$, which confirms the frequency selectivity of the BM.

\section{Parametric Study}

4.1. The Effect of RL. To assess the effect of RL, we remove the $\mathrm{RL}$ from the model, which will result in only axial 
TABle 1: Geometric data of each part of OC.

\begin{tabular}{lcc}
\hline Parameter & Parameter description & Parameter value \\
\hline$b(\mu \mathrm{m})$ & The width of BM & 168 (base) $\sim 200($ apex $)$ \\
$L_{p c}$ & The distance between the left end and the right end of the arch structure & $\mathrm{b} / 3$ \\
$L_{0}$ & The distance between the bottom of the middle row of OHCs and the left end of the BM & b/2 \\
$\alpha$ & The acute angle between OHCs bundles and $z$-axis & $25^{\circ}(\mathrm{base}) \sim 45^{\circ}($ apex $)$ \\
$\beta_{1}, \beta_{2}$ & The acute angle of the arch structure & $\beta_{1}=\beta_{2}=\beta=60^{\circ}$ \\
$\phi$ & The acute angle between OHCs and $z$-axis & $25^{\circ}(\mathrm{base}) \sim 45^{\circ}($ apex $)$ \\
$L_{\text {ohc }}(\mu \mathrm{m})$ & The lengths of OHCs & 70 (base) $\sim 110$ (apex) \\
$L_{\text {ste }}(\mu \mathrm{m})$ & The lengths of OHC bundles & 5 \\
$h_{b m}(\mu \mathrm{m})$ & The thickness of BM & 7 (base) $\sim 1($ apex $)$ \\
$h_{\text {ohc }}(\mu \mathrm{m})$ & The thickness of OHCs & 4.5 \\
$h_{s t e}(\mu \mathrm{m})$ & The thickness of OHC bundles & 0.2 \\
\hline
\end{tabular}

TABle 2: Material properties of the microstructure of OC.

\begin{tabular}{lcc}
\hline Parameter & Parameter description & Parameter value \\
\hline$E_{1}(p a)$ & The elastic modulus of BM & $2 \times 10^{8}($ base $) \sim 1 \times 10^{7}($ apex $)$ \\
$E_{2}(p a)$ & The elastic modulus of OHCs & $4.5 \times 10^{3}($ base $) \sim 1 \times 10^{3}($ apex $)$ \\
$E_{3}(p a)$ & The elastic modulus of OHC bundles & $1 \times 10^{7}($ base $) \sim 1 \times 10^{6}($ apex $)$ \\
$\rho_{b m}\left(\mathrm{~kg} / \mathrm{m}^{3}\right)$ & The density of BM & 1200 \\
$\rho_{\text {ohc }}\left(\mathrm{kg} / \mathrm{m}^{3}\right)$ & The density of OHCs & 1050 \\
$\rho_{s t e}\left(\mathrm{~kg} / \mathrm{m}^{3}\right)$ & The density of OHC bundles & 1200 \\
$v$ & Poisson's ratio of BM & 0.3 \\
\hline
\end{tabular}

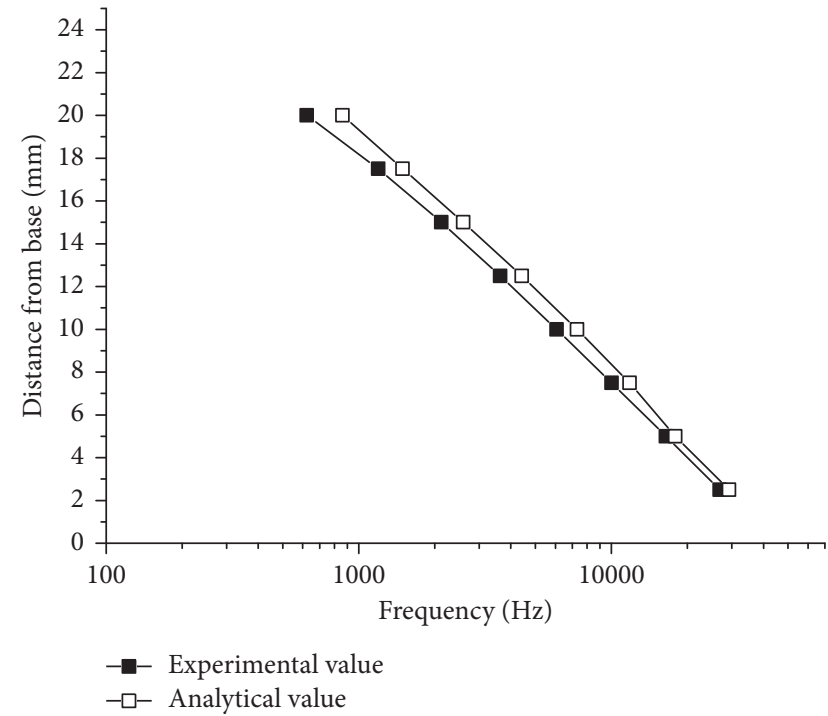

FIgURE 3: Frequency-position relationship of BM.

deformations of the OHC bundles and OHCs during vibration. The frequency response curve is plotted in Figure 5. Figure 5 shows that the amplitude of the response curve decreases significantly, which indicates that the lack of RL causes hearing loss. Moreover, $C F$ seriously deviates from the experimental value.

The reason for these effects is that the stiffness of RL is much greater than that of BM. The force generated by OHCs can be effectively transferred to the BM. At the

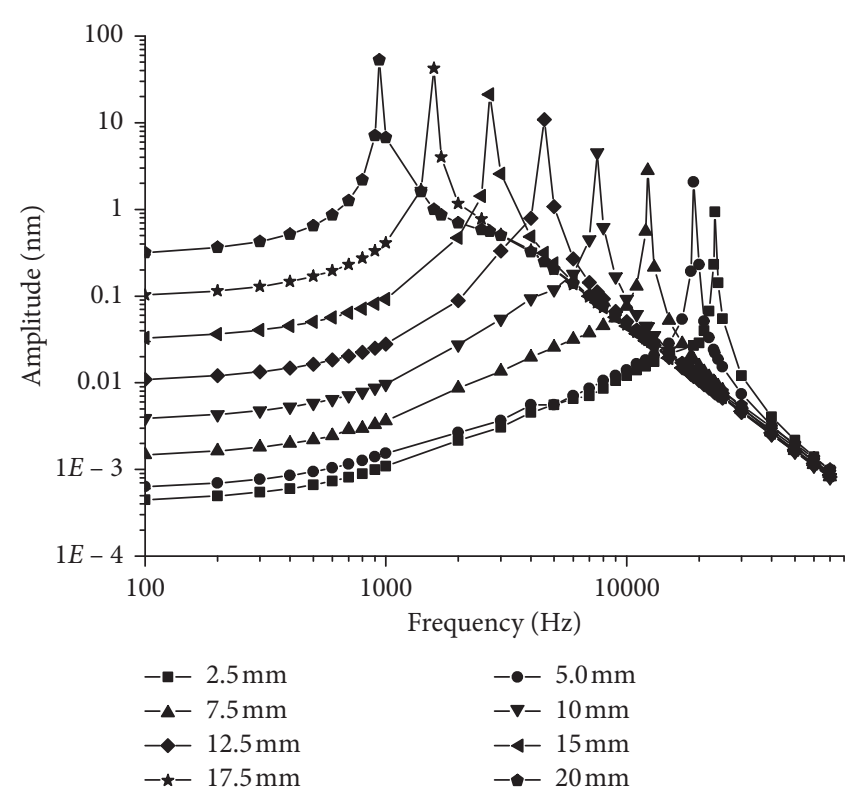

FIGURE 4: Frequency response graph of corresponding points.

same time, a freely moving $\mathrm{RL}$ is highly responsive to the movement of OHCs and enhances the sensitivity of OHCs. It is worth noting that the shear action of the TM and the $\mathrm{RL}$ will cause the opening of ion channels, which is the main reason for producing hearing. Therefore, the RL acts as an effective component in OC to enhance the movement of BM. 


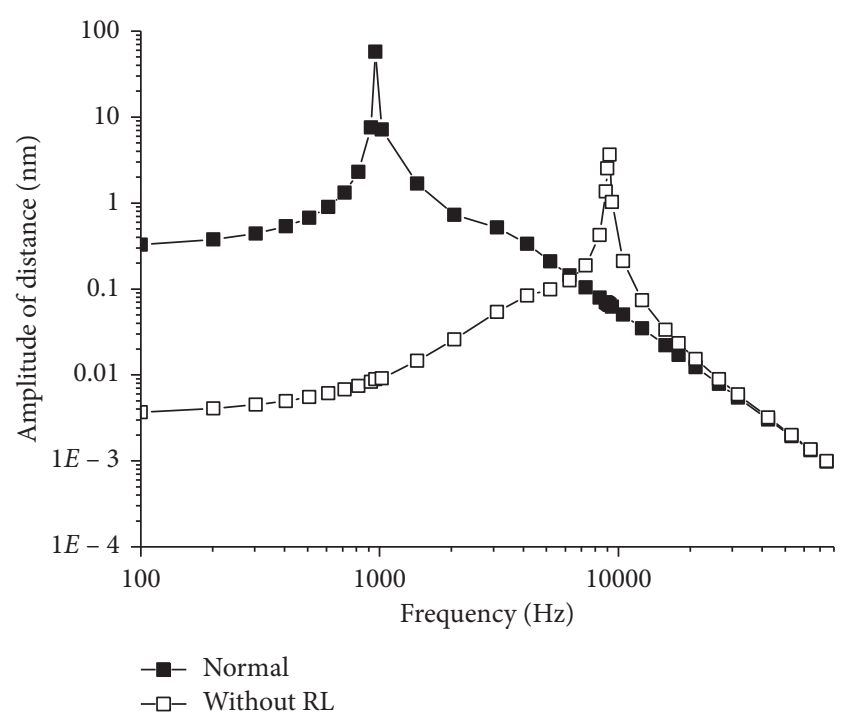

Figure 5: The effect of the lack of RL.

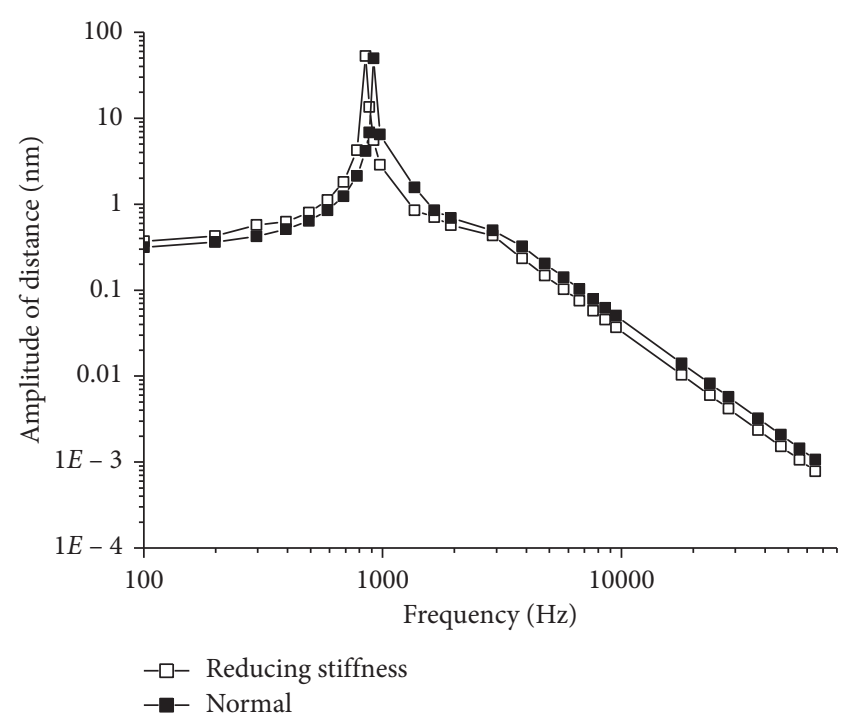

FIgURE 6: The elastic modulus of OHC bundles reducing by half.

4.2. The Effect of OHC Bundles Stiffness. OHC bundles play a significant role in the process of the sound-sensing of the cochlea. A key conclusion is that it has a direct relationship with the generation of the active force [40, 41], and the mechanical properties of $\mathrm{OHC}$ bundles may also have an impact on the tuning of the cochlea. The stiffness of the OHC bundle is an important property because it affects mechanotransduction and, thus, cochlear amplification [42]. Hence, we explore the implications of $\mathrm{OHC}$ bundles by reducing the stiffness to half of the original. The results are presented in Figure 6.

Figure 6 shows that the vibration response curve moves towards the low-frequency direction. The reason for this phenomenon is that reducing $\mathrm{OHC}$ bundles stiffness will reduce the transverse stiffness of OC. At the same time, the

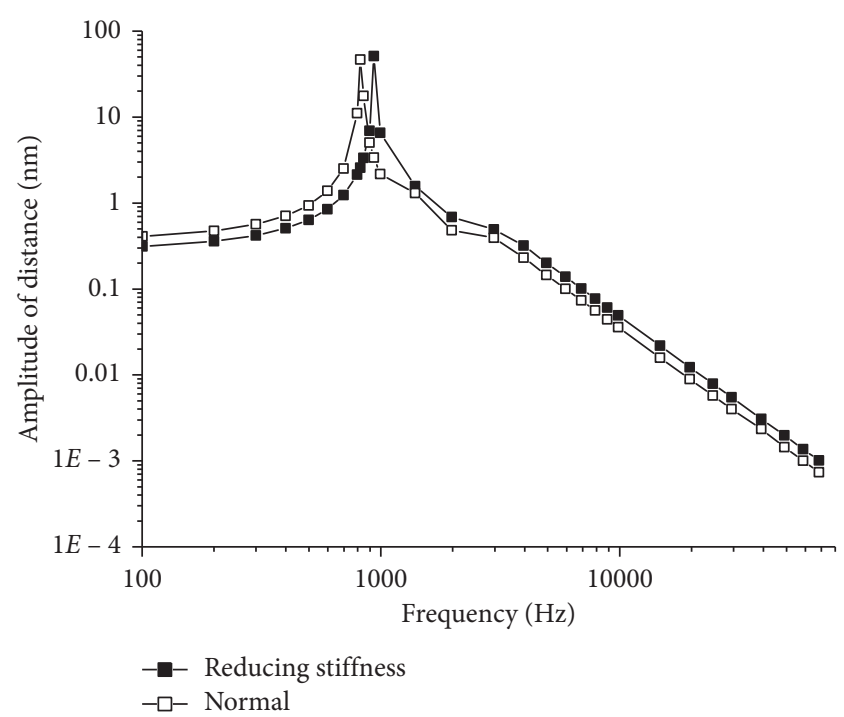

FIgURE 7: The elastic modulus of OHCs reducing by half.

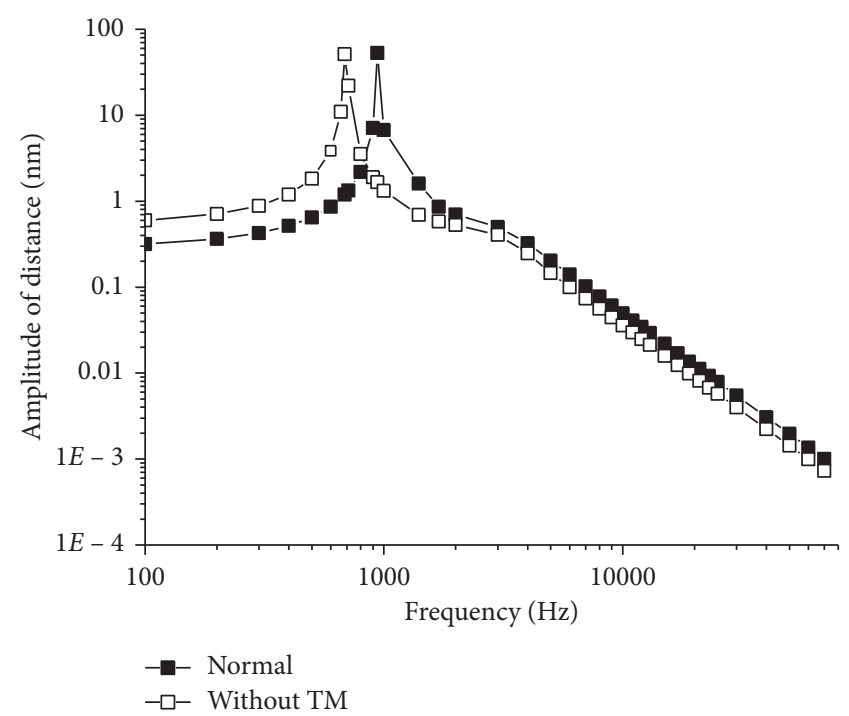

Figure 8: The effect of the lack of TM.

fluctuations of $C F$ value are very little, and the OHC bundles contribute minimally to transverse partition stiffness.

4.3. The Effect of OHCs Stiffness. To explore the role of the stiffness of OHCs, the stiffness of OHCs is reduced by half.

Figure 7 shows that the reduction of OHCs stiffness affects amplitude in different frequency segments. The response curve shifts to lower frequencies, which indicates that the stiffness of the OHCs also contributes to the transverse partition stiffness of cochlea. Moreover, the decrease of $C F$ is slightly above that of $\mathrm{OHC}$ bundles. It is worth noting that the OHCs connect the RL and BM, and they act as a conductive force during the vibration process. 
4.4. The Effect of TM. To assess the impact of TM, establishing a model includes a TM that is exfoliated from the epithelium. Therefore, the analytical expression will not consider the potential energy of the OHC bundles. The results are plotted in Figure 8.

Figure 8 presents that the decrease of $C F$ is more than that of OHC bundles. This finding implies that the TM contributes substantially more to the transverse stiffness of the partition than do the $\mathrm{OHC}$ bundles. In addition, TM directly affects the shear displacement of OHC bundles because it connects $\mathrm{OHC}$ bundles and, thus, cochlear amplification. TM is also an essential part of OC.

\section{Conclusions}

Based on the interaction between BM, OHCs, RL, TM, and so on, a mechanical model of OC is established. The accuracy of the model has been verified by comparing the calculated results with the experimental data. On the basis of the foregoing analysis, the conclusions are expressed by the following.

It is noted that $C F$ of the calculation point seriously deviates from the experimental value when not considering the role of the RL. The amplitude of the frequency-amplitude response curve of the BM decreased significantly, which will cause hearing loss. The RL is an indispensable structure in the process of the sound-sensing mechanism of the cochlea.

The reduction of the stiffness of $\mathrm{OHC}$ bundles and $\mathrm{OHCs}$ will shift vibratory response patterns to lower frequencies. The absence of TM makes $C F$ decrease seriously. These effects are consistent with a reduction in the transverse stiffness of the partition. Significantly, the shear action of the TM and the RL leads to the opening of ion channels at the bottom of $\mathrm{OHC}$ bundles, which strongly affects mechanotransduction. Thus, TM plays an essential role in the process of the sense of sound.

The bottom of the cochlea resonates at high frequencies, and the top resonates at low frequencies. Different points of $\mathrm{BM}$ correspond to different $C F$, which demonstrate the frequency selectivity of the BM. Moreover, the analytical solution of this paper not only reveals the fabric laws of each part of OC but also can be used to carry out parameter sensitivity analysis quickly and effectively. Therefore, it can provide theoretical support for relevant experiments and medical research.

\section{Data Availability}

The data used in this manuscript are available from the corresponding author upon request.

\section{Conflicts of Interest}

The authors declare that they have no conflicts of interest.

\section{Acknowledgments}

The authors acknowledge the support of the Key Project of the National Natural Science Foundation of China (Grant no. 11932010).

\section{References}

[1] L. Robles and M. A. Ruggero, "Mechanics of the mammalian cochlea," Physiological Reviews, vol. 81, no. 3, pp. 1305-1352, 2001.

[2] M. C. Liberman, J. Gao, D. Z. Z. He, X. Wu, S. Jia, and J. Zuo, "Prestin is required for electromotility of the outer hair cell and for the cochlear amplifier," Nature, vol. 419, no. 6904, pp. 300-304, 2002.

[3] W. Brownell, C. Bader, D. Bertrand, and Y. De Ribaupierre, "Evoked mechanical responses of isolated cochlear outer hair cells," Science, vol. 227, no. 4683, pp. 194-196, 1985.

[4] J. Zheng, W. Shen, D. Z. Z. He, K. B. Long, L. D. Madison, and P. Dallos, "Prestin is the motor protein of cochlear outer hair cells," Nature, vol. 405, no. 6783, pp. 149-155, 2000.

[5] H. J. Kennedy, A. C. Crawford, and R. Fettiplace, "Force generation by mammalian hair bundles supports a role in cochlear amplification," Nature, vol. 433, no. 7028, pp. 880-883, 2005.

[6] C. Fernández, "Dimensions of the cochlea (Guinea pig)," The Journal of the Acoustical Society of America, vol. 24, no. 5, pp. 519-523, 1952.

[7] R. Edge, B. N. Evans, and M. Pearce, "Morphology of the unfixed cochlea," Hearing Research, vol. 124, no. 1-2, pp. 1-16, 1998.

[8] R. Glueckert, K. Pfaller, A. Kinnefors et al., "High resolution scanning electron microscopy of the human organ of OC. A study using freshly fixed surgical specimens," Hearing Research, vol. 199, no. 1-2, pp. 40-56, 2005.

[9] K. E. Nilsen and I. J. Russell, "The spatial and temporal representation of a tone on the Guinea pig basilar membrane," Proceedings of the National Academy of Sciences, vol. 97, no. 22, pp. 11751-11758, 2000.

[10] M. Sugawara, Y. Ishida, and H. Wada, "Local mechanical properties of Guinea pig outer hair cells measured by atomic force microscopy," Hearing Research, vol. 174, no. 1-2, pp. 222-229, 2002.

[11] B. F. Zagadou, P. E. Barbone, and D. C. Mountain, "Elastic properties of organ of Corti tissues from point-stiffness measurement and inverse analysis," Journal of Biomechanics, vol. 47, no. 6, pp. 1270-1277, 2014.

[12] H. Cai, B. Shoelson, and R. S. Chadwick, "Evidence of tectorial membrane radial motion in a propagating mode of a complex cochlear model," in Proceedings of the National Academy of Sciences, Oaxaca, Mexico, April 2004.

[13] N. P. Cooper, "Radial variation in the vibrations of the cochlear partition in recent developments in auditory mechanics," World Scientific, vol. 2, pp. 109-115, 2000.

[14] K. E. Nilsen and I. J. Russell, "Timing of cochlear feedback: spatial and temporal representation of a tone across the basilar membrane," Nature Neuroscience, vol. 2, no. 7, pp. 642-648, 1999.

[15] N. P. Cooper and W. S. Rhode, "Fast travelling waves, slow travelling waves and their interactions in experimental studies of apical cochlear mechanics," Auditory Neurosciences, vol. 2, pp. 289-299, 1996.

[16] S. M. Knanna and L. F. Hao, "Reticular lamina vibrations in the apical turn of a living Guinea pig cochlea," Hearing Research, vol. 132, pp. 15-33, 1999.

[17] A. Hubbard, "A traveling-wave amplifier model of the cochlea," Science, vol. 259, no. 5091, pp. 68-71, 1993.

[18] J. B. Allen, "Two-dimensional cochlear fluid model: new results," The Journal of the Acoustical Society of America, vol. 61, no. 1, p. 110, 1977. 
[19] F. Mammano and R. Nobili, "Biophysics of the cochlea: linear approximation," The Journal of the Acoustical Society of America, vol. 93, no. 6, pp. 3320-3332, 1993.

[20] C. R. Steele and L. A. Taber, "Comparison of WKB calculations and experimental results for three-dimensional cochlear models," The Journal of the Acoustical Society of America, vol. 65 , no. 4, p. 1007, 1979.

[21] E. D. Boer, "Nonlinear interactions and the kemp echo," Hearing Research, vol. 2, no. 3-4, pp. 519-526, 1980.

[22] C. D. Geisler, G. K. Yates, R. B. Patuzzi, and B. M. Johnstone, "Saturation of outer hair cell receptor currents causes twotone suppression," Hearing Research, vol. 44, no. 2-3, pp. 241-256, 1990.

[23] D. Manoussaki, R. S. Chadwick, D. R. Ketten, J. Arruda, E. K. Dimitriadis, and J. T. O'Malley, "The influence of cochlear shape on low-frequency hearing," Proceedings of the National Academy of Sciences, vol. 105, no. 16, pp. 6162-6166, 2008.

[24] D. C. Mountain, A. E. Hubbard, and T. A. Mcmullen, "Electromechanical Processes in the Cochlea," Mechanics of Hearing Springer Netherlands, vol. 18, 1983.

[25] H. M. Lieberstein, "The basilar membrane as a uniformly loaded plate clamped on two spiral boundaries in a plane or on two helical-spiral boundaries; discussion of the model," Mathematical Bioences, vol. 12, no. 3-4, pp. 281-291, 1972.

[26] H. M. Lieberstein and J. A. Rickard, "The basilar membrane as a set of uniformly loaded circular annular plates clamped on both edges," Mathematical Bioences, vol. 21, no. 3-4, pp. 339-344, 1974.

[27] J. Ma and W. Yao, "Research on the distribution of pressure field on the basilar membrane in the passive spiral cochlea," Journal of Mechanics in Medicine and Biology, vol. 14, no. 4, pp. 1-22, Article ID 1450061, 2014.

[28] R. Z. Gan, T. Cheng, C. Dai, F. Yang, and M. W. Wood, "Finite element modeling of sound transmission with perforations of tympanic membrane," The Journal of the Acoustical Society of America, vol. 126, no. 1, pp. 243-253, 2009.

[29] X. Wang, L. Wang, J. Zhou et al., "Finite element modelling of human auditory periphery including a feed-forward amplification of the cochlea," Computer Methods in Biomechanics \& Biomedical Engineering, vol. 17, no. 9-12, pp. 1096-1107, 2014.

[30] E. Givelberg and J. Bunn, "A comprehensive three-dimensional model of the cochlea," Journal of Computational Physics, vol. 191, no. 2, pp. 377-391, 2003.

[31] X. Zhang and R. Z. Gan, "A comprehensive model of human ear for analysis of implantable hearing devices," IEEE Transactions on Biomedical Engineering, vol. 58, no. 10, pp. 3024-3027, 2011.

[32] W. J. Yao and J. W. Ma, "Numerical simulation of the human ear and the dynamic analysis of the middle ear sound transmission," Journal of Instrumentation, vol. 8, pp. 1-13, 2013.

[33] W. J. Yao and X. Huang, “Transmitting vibration of artificial ossicle,” Journal of Instrumentation, vol. 9, pp. 131-139, 2008.

[34] Y. Chen and W. Yao, "Numerical simulation on a spiral cochlear," Zhendong Yu Chongii/Journal of Vibration \& Shock, vol. 36, no. 14, pp. 45-51, 2017.

[35] W. Yao, Y. Chen, J. Ma, C. Gan, and D. Wang, "Numerical simulation on the dynamic behavior of the basilar membrane in the spiral cochlea," Biomedical Research (Aligarh), vol. 27, no. 3, pp. 977-984, 2016.

[36] S. Raufer, J. J. Guinan, and H. H. Nakajima, "Cochlear partition anatomy and motion in humans differ from the classic view of mammals," Proceedings of the National Academy of Ences, vol. 116, no. 28, Article ID 201900787, 2019.

[37] S. Elizabeth, "Observing middle and inner ear mechanics with novel intracochlear pressure sensors," Journal of the Acoustical Society of America, vol. 103, pp. 3445-3663, 1998.

[38] D. D. Greenwood, "A cochlear frequency-position function for several species-29 years later," The Journal of the Acoustical Society of America, vol. 87, no. 6, pp. 2592-2605, 1990.

[39] D. D. Greenwood, "Comparing octaves, frequency ranges, and cochlear-map curvature across species," Hearing Research, vol. 94, no. 1-2, pp. 157-162, 1996.

[40] D. Jong, "Estimation of optimal insertion angle in a mammalian outer hair cell stereocilium," Journal of Applied Biomechanics, vol. 45, pp. 1823-1827, 2012.

[41] A. J. Hudspeth, "Integrating the active process of hair cells with cochlear function," Nature Reviews Neuroscience, vol. 15, no. 9, pp. 600-614, 2014

[42] B. D. James and X. Anping, "Mammalian auditory hair cell bundle stiffness affects frequency tuning by increasing coupling along the length of the cochlea," Cell Reports, vol. 23, pp. 2915-2927, 2018. 